\title{
OBITUARY
}

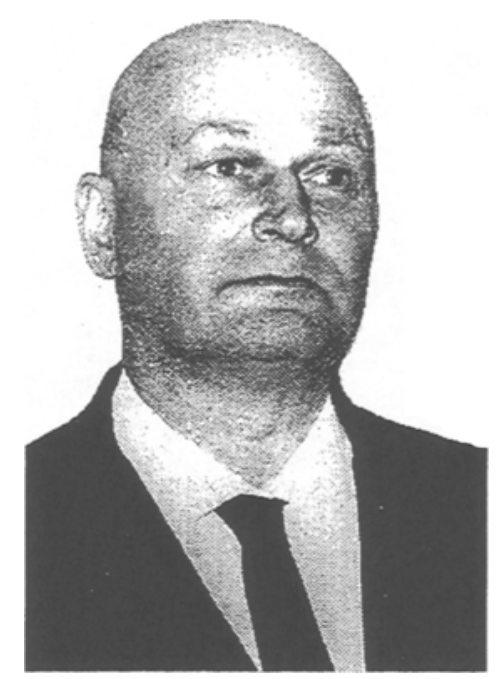

\section{TO THE MEMORY OF VALENTIN ANATOL'EVICH ZMOROVICH}

Ukrainian science suffered an irretrievable loss. Professor of the Chair of Higher Mathematics at the Kiev Polytechnical Institute Valentin Anatol'evich Zmorovich died on February 16, 1994. More than fifty years of his life were devoted to the fruitful teaching and scientific work at the Kiev Polytechnical Institute.

V. A. Zmorovich was born on September 6, 1909 in the family of a school teacher in Kiev. In 1928, he became a student of the Department of Physics and Mathematics of the Kiev Institute of Public Education and graduated from this Institute in 1932. He was formed as a mathematician, scientist, and teacher under a strong influence of Academicians D. A. Grave, N. P. Kravchuk, and N. I. Akhiezer-founders of the Kiev mathematical school. V. A. Zmorovich was still a student when he wrote a chapter to the original textbook in analytic geometry at the request of Prof. D. A. Grave.

The first scientific work of Prof. Zmorovich was published in 1934. In 1937, he defended his Candidate Degree thesis and, in 1950, his Doctor Degree thesis "Studies in the Theory of Analytic and Generalized Analytic Functions". In several years, he became a professor. In 1952-1973, Prof. Zmorovich was the Head of the Chair of Higher Mathematics at the Kiev Polytechnical Institute. Till 1987, he worked as a professor of this Chair.

His lectures, famous for their depth and brilliant presentation, were fascinating not only for students but also for professional teachers.

Many works of Prof. Zmorovich were devoted to the geometric theory of analytic functions. Among the main subjects of his researches into this field were special classes of functions regular and schlicht in a disk, in a circular ring, in an unbounded circular domain, and in $n$-connected circular domains. Zmorovich's method was based on his own generalizations of the classical Poisson, Schwartz, and Riesz-Herglotz integral formulas and their extensions to $n$-connected circular domains. These integral relations can be rightfully named the Zmorovich formulas. His results made it possible to represent many important classes of analytic functions as Stieltjes functionals over a family of nondecreasing functions of bounded variation (or over a collection of families in the case where domains are multiply connected). This technique was called by Prof. Zmorovich the method of structural formulas.

By using this method, he studied the most important qualitative and quantitative characteristics of conformal mappings realized by functions of the indicated types.

With great inherent ingenuity, V. A. Zmorovich constructed the variational calculus of these functionals and applied it to the solution of the following problem: For a fixed point in the domain of definition of functions from a given class, it is necessary to find a function for which the indicated characteristic of a conformal mapping attains an extremal value. Thus, he found the extrema of the curvatures of level lines and their orthogonal trajectories for important classes of analytic functions and indicated the functions for which these extrema are attained. Prof. Zmorovich applied his method to the solution of various mathematical problems. In the literature, it is now named after Zmorovich.

Translated from Ukrainskii Matematicheskii Zhurnal, Vol. 46, No. 8, pp. 1110-1111, August, 1994. 
V. A. Zmorovich also studied generalized analytic functions defined as solutions of elliptic systems of differential equations of a special form. He was also interested in various problems in mathematical analysis, in particular, in the theory of convergence of series.

The inequalities deduced by Prof. Zmorovich were named Zmorovich's inequalities. One of these inequalities was later used in the Belgrade University in constructing efficient quadrature formulas.

Among his scientific and methodical works, one should especially distinguish the book "Practical Manual of the Theory of Definite Integrals," numerous textbooks for students and post-graduate students. In all, he published over 150 works.

Prof. Zmorovich was teaching his students with great love. Since 1950, for almost 40 years, he was at the head of the scientific seminar on the theory of functions and of the scientific and methodical seminar on the general problems in mathematics. These seminars united not only lecturers of the Kiev Polytechnical Institute but also lecturers, researchers, and postgraduate students of many other Ukrainian institutes and universities. V. A. Zmorovich inspired some of his students to the study and further development of important concepts and methods of the geometric theory of functions, namely, of the methods of strips, extremal length, and symmetrization, and their investigations were highly successful. The 50s and 60 s were the most fruitful years of his pedagogic career. Prof. Zmorovich was the supervisor of fifteen successfully defended Candidate Degree theses. Three of his disciples (L. A. Dunduchenko, I. P. Mityuk, and P. M. Tamrazov) became Doctors of Sciences. Many students Prof. Zmorovich are now professors, some of them are at the head of mathematical chairs at many Ukrainian institutes and universities.

V. A. Zmorovich was always a responsible, humane, and honest person, a man of principle. His high moral standards were brightly exhibited in his attitude to the teacher, academician M. P. Kravchuk, who was arrested and convicted without any grounds in 1938. He and his wife R. I. Demakhovskaya (at that time, she was an assistant of Prof. Kravchuk) never joined the choir of accusations. They were always sure that he was innocent and did not make any secret of their position.

For his productive scientific and teaching work, Prof. Zmorovich was decorated with two Orders "Sign of Honor" and many medals.

The memory of Valentin Anatol'evich Zmorovich, a prominent scientist, talented teacher, humane and benevolent man, a man of principle, will always live in our hearts.

F. T. Baranovskii, Yu. M. Berezanskii, V. V. Buldygin, Yu. L. Daletskii, V. K. Dzyadyk, V. A. Dobrovol'skii, V. G. Lozovik, Yu. A. Mitropol'skii, A. M. Samoilenko, I. V. Skrypnik, P. M. Tamrazov, F. P. Yaremchuk 